\title{
Lymphoid-specific helicase in epigenetics, DNA repair and
}

\section{cancer}

\author{
Xiangyu Chen ${ }^{1,2}$, Yamei $\mathrm{Li}^{1,2}$, Karla Rubio $\mathbb{D}^{3,4,5,6}$, Bi Deng ${ }^{1,2}$, Yuyi $\mathrm{Li}^{1,2}$, Qinwei Tang ${ }^{1,2}$, Chao Mao $\mathbb{D}^{1,2}$, Shuang Liu ${ }^{7}$, Desheng Xiao ${ }^{1,2}{ }^{\bowtie}$, \\ Guillermo Barreto $\mathbb{D}^{3,4,5,6 \bowtie}$ and Yongguang Tao (iD ${ }^{1,2,8}$
}

(C) The Author(s), under exclusive licence to Springer Nature Limited 2021

Lymphoid-specific helicase (LSH) is a member of the SNF2 helicase family of chromatin-remodelling proteins. Dysfunctions or mutations in LSH causes an autosomal recessive disease known as immunodeficiency-centromeric instability-facial anomaly (ICF) syndrome. Interestingly, LSH participates in various aspects of epigenetic regulation, including nucleosome remodelling, DNA methylation, histone modifications and heterochromatin formation. Further, LSH plays a crucial role during DNA-damage repair, specifically during double-strand break (DSB) repair, since murine LSH was shown to be essential for non-homologous end joining (NHEJ) and homologous recombination (HR). Accordingly, overexpression of LSH drives tumorigenesis and malignancy. On the other hand, LSH homologs stabilise the genome. Thus, LSH might be implemented as a biomarker for various cancer types and potential target molecule to develop therapeutic strategies against them. In this review, we focus on the role of LSH in orchestrating chromatin rearrangements, such as DNA methylation and histone modifications, as well as in DNA-damage repair. Changes in chromatin structure may facilitate gene expression signatures that cause malignant transformation. We summarise recent findings of LSH in cancers and raise critical open questions for further studies.

British Journal of Cancer; https://doi.org/10.1038/s41416-021-01543-2

\section{BACKGROUND OF LSH}

Epigenetic mechanisms of transcriptional regulation involve DNA methylation, histone modifications, nucleosome positioning and heterochromatin formation [1], all of which contribute to establishing of specific gene expression signatures resulting in lineage-specific cell fates [2,3]. Epigenetic changes of chromatin are heritable and reversible, being written, read and erased by a vast number of proteins belonging to different protein superfamilies, including ATP-dependent chromatin-remodelling complexes, which either move, eject or restructure nucleosomes. There are at least four families of chromatin remodelers in eukaryotes: SWI/SNF, ISWI, NuRD/Mi-2/CHD and INO80 [4]. Although all chromatin-remodelling complexes share a common ATPase domain, their functions are specific in the context of different biological processes. LSH belongs to the SNF2 helicase family from the helicase-like superfamily 2 (SF2). Within SF2, the SNF2 family refers to proteins with homologous sequence to the Saccharomyces cerevisiae Snf2p. The SNF2 family consists of a large group of ATP-hydrolysing proteins, which are generally present in eukaryotes, as well as eubacteria and archaea [5]. They are ATPdependent chromatin remodelers that facilitate the accessibility of DNA to transcription factors [6, 7]. Members of the SNF2 family share a common core of two recA-like domains, and link ATP hydrolysis to a change in the relative orientation of these domains [5].

The murine LSH homolog is also named PASG and SMARCA6 [8]. Jarvis et al. found that the Lsh gene is required for lymphocyte development and immunoglobulin class switch recombination [9]. A further study by Geiman et al. demonstrated that $L s h$ is required for normal murine development [10]. Moreover, Lsh is required for genome-wide methylation since its depletion led to genome-wide hypomethylation [11-13]. Interestingly, Lee et al. revealed that the levels of the human LSH homolog, named HELLS, were reduced after cytokine withdrawal in the human acute megakaryoblastic leukaemia cell line, MO7e, whereas HELLS levels were increased in highly proliferative tissues, indicating its association with cellular proliferation [8]. Interestingly, the impact of LSH on cellular proliferation is demonstrated in tumour progression, as failure to

\footnotetext{
${ }^{1}$ Department of Pathology, Xiangya Hospital, Central South University; Key Laboratory of Carcinogenesis and Cancer Invasion, Ministry of Education, Hunan, China. ${ }^{2}$ NHC Key Laboratory of Carcinogenesis of Ministry of Health (Central South University); Cancer Research Institute, Central South University, Changsha, Hunan, China. ${ }^{3}$ Université de Lorraine, CNRS, Laboratoire IMoPA, UMR 7365, Nancy, France. ${ }^{4}$ Univ Paris Est Creteil, Gly-CRRET, Brain and Lung Epigenetics (BLUE), Creteil, France. ${ }^{5}$ Lung Cancer Epigenetic, MaxPlanck-Institute for Heart and Lung Research, Bad Nauheim, Germany. ${ }^{6}$ International Laboratory EPIGEN, Universidad de la Salud del Estado de Puebla, Puebla, Mexico. ${ }^{7}$ Department of Oncology, Institute of Medical Sciences, National Clinical Research Center for Geriatric Disorders, Xiangya Hospital, Central South University, Changsha, Hunan, China. ${ }^{8}$ Department of Thoracic Surgery, Hunan Key Laboratory of Early Diagnosis and Precision Therapy in Lung Cancer and Second Xiangya Hospital, Central South University, Changsha, China. ${ }^{\circledR}$ email: xdsh96@csu.edu.cn; Guillermo.barreto@univ-lorraine.fr; taoyong@csu.edu.cn
} 
repress $L s h$ in retinoblastoma mouse models resulted in retinal tumorigenesis [14]. More recently, the function of LSH has broadened into DNA-damage response (DDR) regulation and carcinogenesis, which will be further discussed below. Considering that LSH acts as an upstream regulator in the network driving a tumorigenic phenotype, it might be considered a potential target for future therapies.

\section{LSH is a key regulator of chromatin structure}

Pluripotency of embryonic stem cells strongly relies on specific gene expression signatures which are partially determined by their chromatin structure $[15,16]$. ATP-dependent chromatin-remodelling complexes are fundamental regulators of chromatin and gene expression, including members of the four families of chromatin remodelers: SWI/SNF, ISWI, NuRD/Mi-2/CHD and INO80. Complexes of the SWI/SNF family activate promoter/enhancer regions by epigenetic modulation of chromatin structure. LSH, a chromatinremodelling protein of the SNF2 family, is primarily responsible for ATP-dependent nucleosome remodelling $[17,18]$. In line with this idea, Ren et al. showed that $L s h$ is required for proper nucleosome density implementing nucleosome occupancy assays in wild-type (wt) and Lsh-deficient (Lsh-/-) embryonic stem (ES) cells [17]. In addition, by integrative analysis of RNA sequencing and micrococcal nuclease sequencing in hepatocellular carcinoma (HCC) cells, Law et al. revealed that overexpression of HELLS (gene coding for human LSH) increased nucleosome occupancy. This obstructed the accessibility of enhancers and hindered the formation of the nucleosome-free region at the transcription start sites. These events resulted in epigenetic silencing of multiple tumour suppressor genes and HCC progression [18].

Furthermore, LSH is also involved in other mechanisms affecting chromatin structure, such as DNA methylation and histone modifications (Fig. 1). DNA methylation is the best-characterised DNA modification and occurs mainly at carbon 5 in the cytosine (5-methylcytosine, 5mC) of CpG dinucleotides in eukaryotes $[19,20]$. Proper DNA methylation patterns are essential for cell differentiation and embryonic development. DNA methylation plays a critical role in gene repression and genome stability by preventing recombination events between repetitive sequences [20]. DNA methylation is mediated by DNA methyltransferases (DNMTs), including DNMT1, DNMT3A and DNMT3B [21]. Interestingly, DNA methylation at repeat elements, a hallmark of heterochromatin, is significantly reduced in Lsh-/- ES cells [17]. Moreover, the ATP binding site of LSH is required for stable association of DNMT3B to repetitive elements, supporting that the nucleosome remodelling activity of LSH on chromatin is crucial for DNA methylation at repetitive elements and, consequently, for heterochromatin formation [17]. In addition, the methylation of satellite sequences requires CDCA7, which facilitates the recruitment of LSH onto chromatin [22]. According to these findings, fibroblasts derived from $L s h-/-$ mouse embryos, which lack DNA methylation from centromeric repeats, transposons and several genes' promoters, are capable of reestablishing DNA methylation pattern after re-expression of $L s h$, thereby demonstrating the causal involvement of LSH in methylation of repetitive elements and heterochromatin formation [23]. Further, Lsh is also required for methylation of transposable elements, mitosis and meiosis during cell division and gametogenesis, respectively, as demonstrated by defects detected in Lsh-/ - mice during meiotic chromosome synapsis in female germ cells, centromere transcription during oocytes meiosis and meiotic progression in spermatocytes [24-27]. Interestingly, LSH has been shown to regulate long terminal repeats retrotransposon repression independent of DNMT3B [28]. Dunican et al. propose a model in which Lsh is required at a specific time window during development to target de novo methylation to repetitive sequences, which DNMT1 subsequently maintains to enforce selective silencing of the targeted repetitive sequences [28].
DNA methylation can be reverted back via active DNA demethylation, which consists of a series of oxidation steps converting $5 \mathrm{mC}$ to 5-hydroxymethylcytosine (5-hmC), 5 -formylcytosine $(5 \mathrm{fC})$, and 5-carboxylcytosine $(5 \mathrm{caC})$. The latter two are eventually excised by thymine DNA glycosylase (TDG), followed by base-excision repair (BER) to restore the cytosine $[29,30]$. Conversion of $5 \mathrm{mC}$ to 5 -hmC by ten-eleven translocation (TET) family enzymes plays an essential biological role in embryonic stem cells, development, ageing and disease [31]. Interestingly, LSH has been shown to interact with TET2 and participates in the conversion of $5 \mathrm{mC}$ to 5 -hmC specifically at heterochromatin-associated satellite DNA loci [32]. Moreover, LSH promoted genome stability by silencing satellite expression, affecting 5-hmC levels in pericentromeric satellite repeats. Decreased levels of LSH in specific cancer types are one of the mechanisms underlying 5 -hmC reduction, genome instability and metastasis [32]. Due to the function of TETs as mediators of active DNA demethylation [31], the interaction between TET2 and LSH seems to be paradoxical at first instance. However, previous studies indicated that loss of TET is partially associated with genome hypomethylation, while DNMT3A and DNMT3B facilitate DNA demethylation at low concentrations of the methyl group donor, S-adenosyl methionine [33]. Thus, elucidating the unconventional roles of TETs and DNMTs, and how LSH impacts DNA methylation in cooperation with TETs and DNMTs remains on the scope.

Besides DNA methylation, LSH is also involved in histone modifications. While DNA methylation is a relatively stable change in somatic cells, post-translational modifications of histone proteins are more diverse and complex, and can change rapidly during the cell cycle [19]. Histone proteins are part of nucleosomes, which are the structural and functional units of chromatin. Histone proteins can undergo post-translational modifications at their $\mathrm{N}$-terminal tails, including acetylation, methylation, phosphorylation, ubiquitination and sumoylation, among others [34-36]. Specific combinations of histone modifications called the "histone code" imparts the expression status of a region of the chromatin [34]. It seems that Lsh is required for normal histone methylation, since in Lsh-/- mouse embryonic fibroblasts (MEFs) the reduction of DNA methylation is associated with de novo monomethylation of the core histone 3 at lysine 4 (H3K4me1) [37]. Interestingly, $\mathrm{Yu}$ et al. showed a functional correlation between $\mathrm{H} 3 \mathrm{~K} 4 \mathrm{me} 1$ enrichment at genomic sites with reduced DNA methylation, enhancer formation and cellular plasticity by characterising induced pluripotent stem (iPS) cells from wt and Lsh-/- MEFs [37]. Moreover, Lsh knockout results in accumulation of di- and tri-methylated histone 3 at lysine 4 (H3K4me2 and H3K4me3) at repetitive sequences, including pericentromeric DNA [12], whereas di- or tri-methylation of $\mathrm{H} 3$ at lysine 9 (H3K9me2 and H3K9me3) appear unchanged after $L s h$ deletion [12]. On the other hand, LSH gain-of-function (GOF) increases the repressive histone mark $\mathrm{H} 3 \mathrm{~K} 27 \mathrm{me} 3$ and decreases the active histone mark H3K4me3, supporting that LSH mediates gene repression upon binding to promoters [18, 38]. Interestingly, LSH-mediated histone methylation seems essential for cellular plasticity and differentiation of ES cells or MEFs under conditions favouring specific lineage differentiation [37, 39]. Ren et al. showed that the association of LSH to the Oct4 promoter during differentiation of ES cells mediates transcriptional repression, which is accompanied by less chromatin accessibility, an increase of repressive histone marks and gain of DNA methylation at distal and proximal Oct4 enhancer sites [38]. Following this line of ideas, $\mathrm{Xi}$ et al. demonstrated that LSH associates to some Hox genes during embryonic development and mediates their silencing by regulating DNMT3B binding, DNA methylation and specific repressive histone modifications, the last ones through the interaction with components of the polycomb repressive complex 1 (PRC1) [40-42]. 


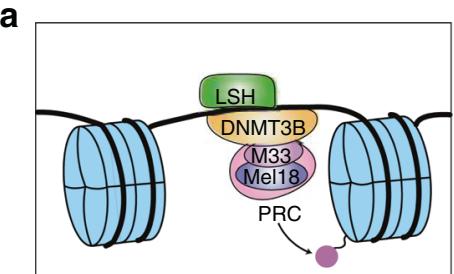

Hox gene

C

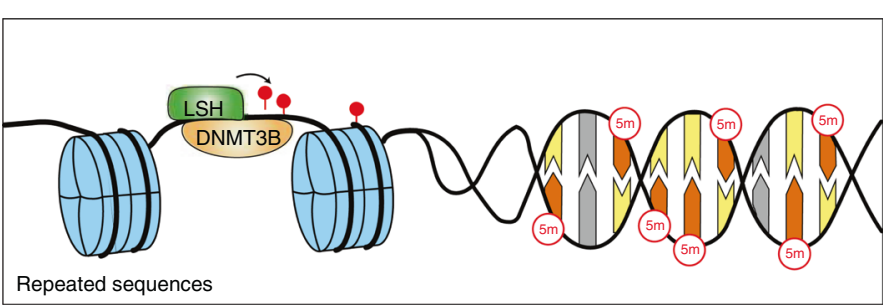

d

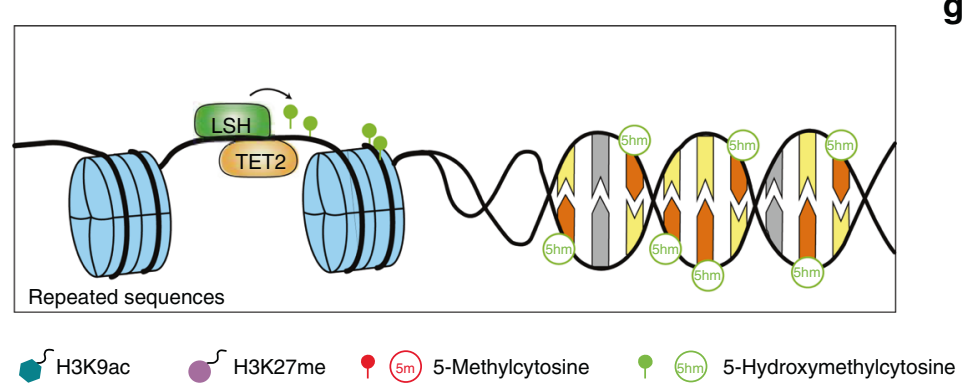

b

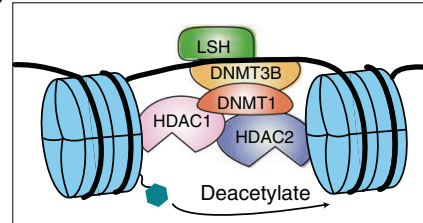

Upstream of a reporter gene e

f
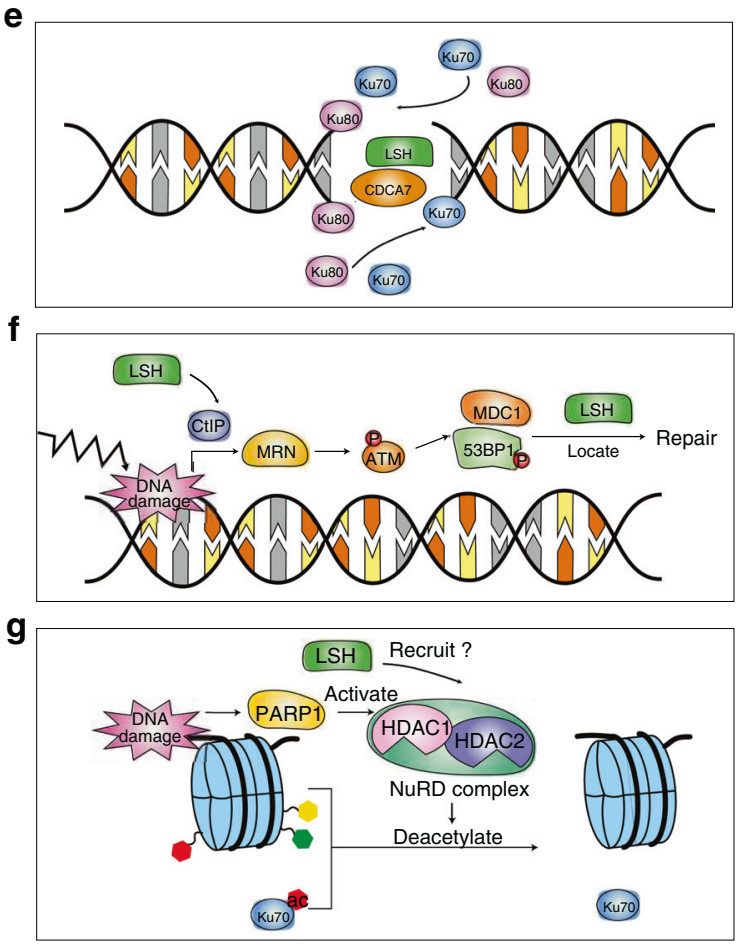

Cytosine $\triangle$ Guanine $\mathrm{N}$ H3K56ac of H4K16ac o H4K91ac

Fig. 1 LSH is a key regulator of chromatin structure and DNA-damage repair. a LSH interacts with DNMT3B and PRC components to promote H3K27 methylation of Hox genes and therefore silences their expression during cell development. $\mathbf{b}$ LSH recruits DNMT3B, DNMT1, HDAC1 and HDAC2 to deacetylate H3K9ac, acting as a histone transcriptional repressor. c LSH participates in the methylation of repetitive sequences, which are essential components of heterochromatin. LSH helps DNMT3B locate to DNA and methylate centromeric repeats, transposons and gene promoters rich with CpG sites. d LSH upregulates TET2 and forms a complex with it to maintain the genomic 5-hmC level. In response to DNA-damage, mammalian cells initiate DDR and downstream pathways. For DSB, there are two major pathways to repair the damage, NHEJ and HR. Besides, two minor pathways, rd-NHEJ and MMEJ, can ligate ends. As a member of the SWI/SNF chromatin remodeler family, LSH helps to remodel chromatin and facilitates repairing molecules that binds to damaged sites. e LSH and CDCA7 participate in C-NHEJ, in which they recruit protein Ku70 and Ku80 to the damaged sites for afterwards repairing. $\mathbf{f}$ In HR, after DNA damage, MRN and ATM are activated, impacting various substrates. LSH recruits MDC1 and 53BP1 protein to targeted sites, facilitating the repairing process. $g$ In MMEJ, HDAC1 and HDAC2 participate in DNA repair by deacetylating H3K56, H4K16 and H4K91, as well as Ku70. Considering that LSH cooperates with HDAC1 and HDAC2 in epigenetic modulation of histone, there exists a possibility that LSH recruits HDAC1 and HDAC2 in response to DNA damage.

Along with methylation of histones, LSH also facilitates deacetylation of histones. LSH cooperates with DNMT1, DNMT3B and the histone deacetylases (HDACs) HDAC1 and HDAC2 to reduce acetylation levels of $\mathrm{H} 3$ and $\mathrm{H} 4$ and to silence transcription [43]. For instance, when this complex is targeted to GAL4-binding sites upstream of a reporter gene promoter, it represses gene expression by deacetylating $\mathrm{H} 3 \mathrm{~K} 9 \mathrm{ac}$ and H4K12ac [43]. Myant and Stancheva proposed that LSH serves as a recruiting factor for DNMTs and HDACs to establish transcriptionally repressive chromatin that is further stabilised by DNA methylation at specific loci [43].

In conclusion, LSH is involved in various aspects of epigenetic gene silencing, including chromatin remodelling, DNA methylation and histone modifications, which in turn are crucial for different biological processes, such as pluripotency, somatic cell reprogramming, embryonic development, cell differentiation, as well as pathological processes including malignant transformation and tumour progression, among others.

\section{LSH acts as a modulator of DNA repair}

DNA damage is usually caused by exposure to genotoxic agents and physiological DNA transitions. Single-strand DNA (ssDNA) breaks are DNA lesions typically resulting from oxidative damage or base hydrolysis. ssDNA breaks lead to substitutions or even DSB. Other situations, such as depurination, depyrimidination and
8-oxoG, occur frequently and determine the standard organisation of DNA [44, 45]. DSB, one of the most severe lesions, arises from ionising radiation, reactive oxygen species, DNA replication errors and inadvertent cleavage by nuclear enzymes [46]. Further, DSB shows a high incidence at repetitive DNA, non-B DNA structures, DNA-protein barriers and highly transcribed regions [46].

To preserve genomic integrity, eukaryotic cells develop DDR. In response to DSB, DDR initiates two downstream pathways: nonhomologous end joining (NHEJ) and homologous recombination (HR) $[47,48]$. NHEJ restores DNA integrity in an error-prone way since it could ligate two ends without any deletion or addition from either strand. As for HR, a homologous DNA molecule is requested as a template; therefore, $\mathrm{HR}$ is generally error-free and activated during $S$ and $G 2$ phases [49]. Although LSH does not repair DNA damages directly, recent studies depicted LSH as a positive modulator in DSB repair, both in canonical NHEJ (C-NHEJ) and $H R$, unveiling its impacts on genomic homoeostasis and cancer biology (Fig. 1) [45].

C-NHEJ is initiated by protein Ku80 (XRCC5 or Ku86) and Ku70 (XRCC6), which bind to DSB ends and recruit DNA-dependent protein kinase catalytic subunit $[50,51]$. Once started, DNA ligase IV complex, including DNA-dependent protein kinase, ligase IV, $X$-ray repair cross-complementing gene 4 (XRCC4) and XRCC4-like factor will ligate ends and multiple substrates will be phosphorylated to complete the repairing [50]. In C-NHEJ, LSH helps to 
recruit Ku80 and Ku70 to DSB sites. Dysfunction of LSH protein leads to ICF type 4, while mutations in DNMT3B, ZBTB24 and CDCA7 genes are responsible for ICF types 1, 2 and 3. All four ICF types show phenotypic similarities, including DNA hypomethylation and immune deficiencies [45]. The function of LSH, DNMT3B, ZBTB24 and CDCA7 is correlated with each other. Whereas ZBTB24 enhances the transcription of the CDCA7 gene, the CDCA7 protein promotes the assembly of LSH onto chromatin. Further, LSH recruits DNMT3B and DNMT3B mediates DNA methylation [22]. Malfunction of any protein of the ZBTB24-CDCA7-LSH-DNMT3B axis leads to DNA methylation defects and vulnerability to DNA damage. Mutations in ICF-related genes, including ZBTB24, CDCA7,
LSH and DNMT3B, affect cellular DNA-repair ability, and therefore, increase the levels of the DNA-damage marker $\mathrm{\gamma H} 2 \mathrm{AX}$, particularly in the centromeric, pericentromeric, and telomeric regions [45]. Interestingly, a recent study found that the C-NHEJ pathway was altered in Lsh-deficient B cells in a Ku70/80 independent manner, indicating that LSH might participate in C-NHEJ by different mechanisms [52].

In addition to C-NHEJ, LSH participates in HR [53]. In response to DSB damage, HR is embarked with break recognition and endresection of the MRE11-RAD50-NBS1 (MRN) complex in association with CtIP $[54,55]$. Next, MRN interacts with ataxia-telangiectasia mutated (ATM) kinase or directly activates ATM, as they both a
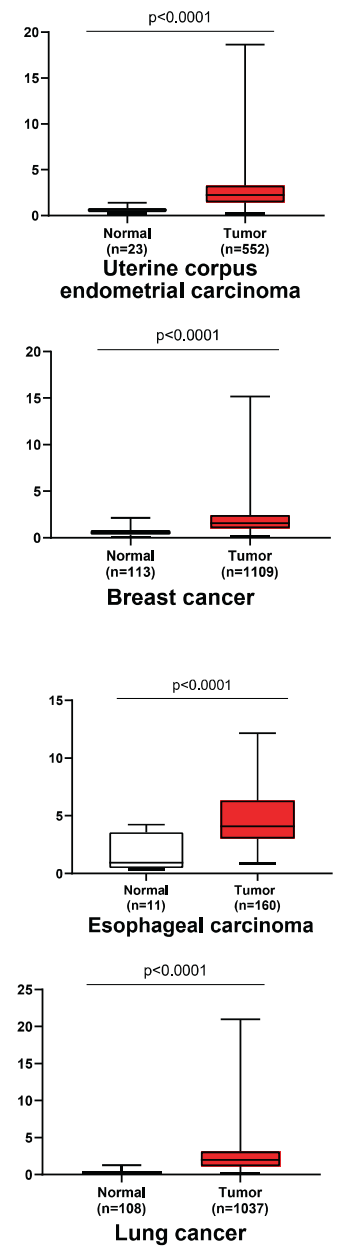
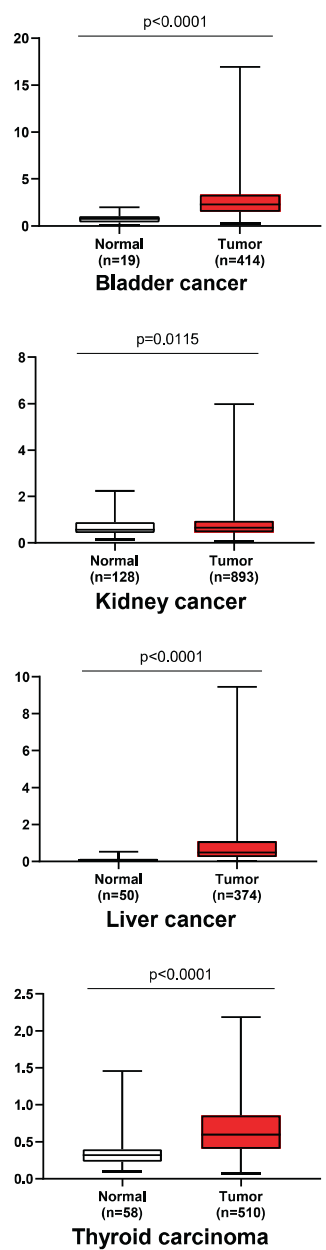

b

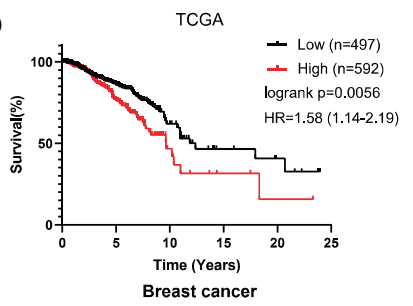

TCGA

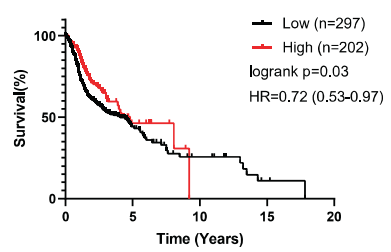

Head-neck squamous cell carcinoma

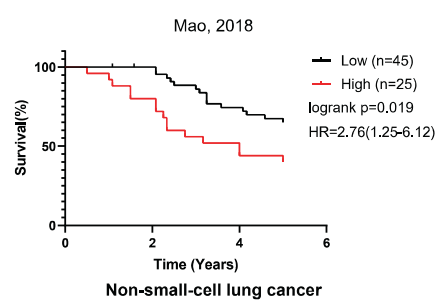

TCGA

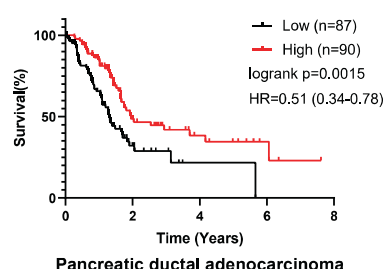

TCGA

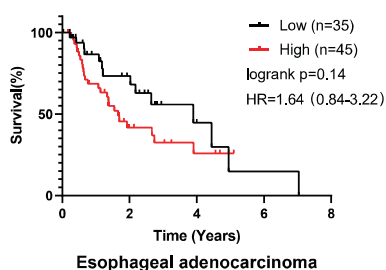

TCGA

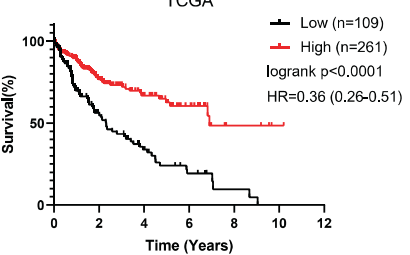

Liver hepatocellular carcinoma

TCGA

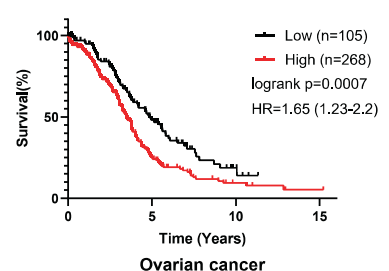

Ovarian cancer

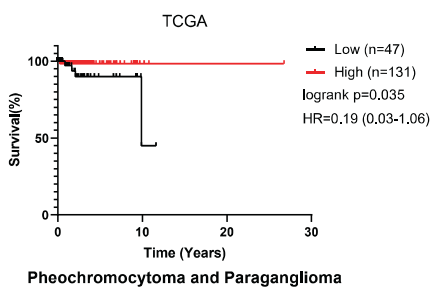

Fig. 2 The expression of LSH in various cancers. Data analysed in this figure were extracted from the TCGA database (https://portal.gdc. cancer.gov/). The overall survival rate graph of NSCLC is from the study conducted by Mao et al., and the usage of the graph was approved (PMID: 30094095) [95]. a LSH is overexpressed in many types of cancers. Here displays mRNA level in several kinds of cancers with the statistical difference $(P<0.05)$ between cancerous samples and normal ones. Information on LSH mRNA expression is from TCGA. To conduct the overall analysis, we filtered data with restriction, including transcriptome profiling of data category, gene expression qualification of data type, RNA-seq of experimental strategy and HTSeq-FPKM of workflow type. The nine presented graphs and the related projects are (bladder cancer: TCGA-BLCA), (breast cancer: TCGA-BRCA), (oesophageal carcinoma: TCGA-ESCA), (kidney cancer: TCGA-KIRC, TCGA-KIRP and TCGA$\mathrm{KICH}$ ), (liver cancer: TCGA-LIHC), (lung cancer: TCGA-LUAD and TCGA-LUSC), (thyroid carcinoma: TCGA-THCA) and (uterine corpus endometrial carcinoma: TCGA-UCEC). The gene expression levels were computed in an unpaired t-test using GraphPad Prism (v8.0.2). b The survival time correlates with the expression level of LSH. There is a correlation between patients' survival time and the mRNA level of LSH. The elevated level of LSH mRNA could be a risk factor in breast cancer, oesophageal carcinoma, NSCLC and ovarian cancer. In contrast, high level of LSH mRNA might be a protective factor in head-neck squamous cell carcinoma, liver hepatocellular carcinoma, pancreatic ductal adenocarcinoma and pheochromocytoma and paraganglioma. Data were respectively extracted: breast cancer from TCGA-BRCA, oesophageal carcinoma from TCGA-ESCA, NSCLC from the study conducted by Mao et al. [95], ovarian cancer from TCGA-OV, head-neck squamous cell carcinoma from TCGA-HNSC, liver hepatocellular carcinoma from TCGA- LIHC, pancreatic adenocarcinoma from TCGA-PAAD, and pheochromocytoma and paraganglioma from TCGA-PCPG. Overall survival Kaplan-Meier estimate was completed based on public TCGA data with GraphPad Prism (v8.0.2) and default settings. The outcomes demonstrate that the correlation can be positive or negative, depending on types of cancers. Only curves with $P<0.05$ are displayed. 
assemble at the DSB sites [56]. Along this cascade, many effectors are activated. For example, after being activated by ATM, the mediator of DNA-damage checkpoint 1 (MDC1) recruits ATM to DSB sites, promoting the process [57]. Afterwards, with the phosphorylation at threonine 68 (T68) by ATM and MDC1, DDR checkpoint kinase 2 (CHK2) is activated and arrests the cell cycle [58]. Furthermore, P53-binding protein 1 (53BP1), a dispensable player in the HR process, is dephosphorylated via a BRCA1dependent mechanism, contributing to HR by relaxing densely packed heterochromatin [59]. In this network, LSH recruits MDC1 and $53 \mathrm{BP} 1$ to the damage sites and facilitates the HR process of heterochromatin by facilitating end-resection and accumulation of CtIP at IR-induced foci $[53,60]$. In the aspect of DSB induced within heterochromatin, downregulation of LSH results in fewer ssDNA foci, which partially overlaps with the defect in CtIP-depleted cells, indicating that LSH facilitates efficient repair of breaks [53]. In addition, LSH helps to open chromatins before meiosis. It is recruited by PRDM9 to HR hot spots, where most recombination occurs, and facilitates histone modifications enhancing chromatin accessibility [61].

Apart from Ku80, Ku70, MDC1, 53BP1 and CtIP, other proteins associated with $\mathrm{LSH}$ and DSB repair pathways have been suggested. Resection-dependent NHEJ (rd-NHEJ) and microhomology-mediated end joining (MMEJ) are two alternative DSB repair pathways that act independently from Ku70/80 [54]. In MMEJ, the NuRD complex is recruited to rebuild the normal DNA structure [62]. In the NuRD complex, HDAC1 and HDAC2 promote DSB repair by deacetylating H3K56ac, H4K16ac and H4K91ac [6365]. Also, HDAC1 and HDAC2 deacetylate Ku70 [66]. Inhibition of HDAC1 and HDAC2 in prostate cancer cells resulted in increased levels of Ku70 acetylation decreased Ku70 binding to DSB sites, and hypersensitivity to DSB induced by chemotherapy [66]. Though no direct evidence shows that LSH recruits HDAC1 and
HDAC2 to the damage sites, a substantial probability of LSHmediated recruitment of HDAC1 and HDAC2 to DNA exists.

To sum up, despite no direct function on DNA repair, LSH plays a significant role as a mediator of DNA-damage repair by recruiting other proteins, particularly during DSB. From this perspective, LSH handles the proper assembly of repairing proteins, cell-cycle controlling proteins, and possibly recruits HDACs to DSB sites to maintain genomic stability. However, since most experiments were conducted in mouse cells, further validation of the presence of the same molecular mechanisms in human cells remains essential.

\section{LSH is a bridge regulator in oncogenesis}

The increase of LSH protein and mRNA has recently emerged as a hallmark in many cancer types (Fig. 2). Clinical data extracted from the TCGA database demonstrated that elevated $L S H$ mRNA levels negatively correlate with patients' overall survival in several kinds of cancers, including breast cancer, oesophageal adenocarcinoma, non-small-cell lung cancer and ovarian cancer (Fig. 2). Whereas in other cases, the aberrant levels of $L S H$ mRNA could be a protective factor (Fig. 2) [67-69]. In non-small-cell lung carcinoma (NSCLC), benzo(a)pyrene (BaP), a component of polycyclic aromatic hydrocarbons derived from smoke and air pollution, recruits the aryl hydrocarbon receptor $(\mathrm{AhR})$ to the promoter of the $L S H$ gene and upregulates the levels of the LSH protein [70]. In hepatocellular carcinoma (HCC), the specificity protein 1 (SP1) elevates the expression of $L S H$ by binding the upstream TSS [18]. In head and neck squamous cell carcinoma, the mRNA levels of LSH positively correlate with the transcription of the critical oncoprotein, forkhead box M1 (FOXM1) [69]. Degradation of LSH is suppressed in these cancer subtypes since its interaction with ubiquitin C-terminal hydrolase L3 (UCHL3), a member of the deubiquitinating enzyme family, is interfered by the IncRNA GIAT4RA [71]. In

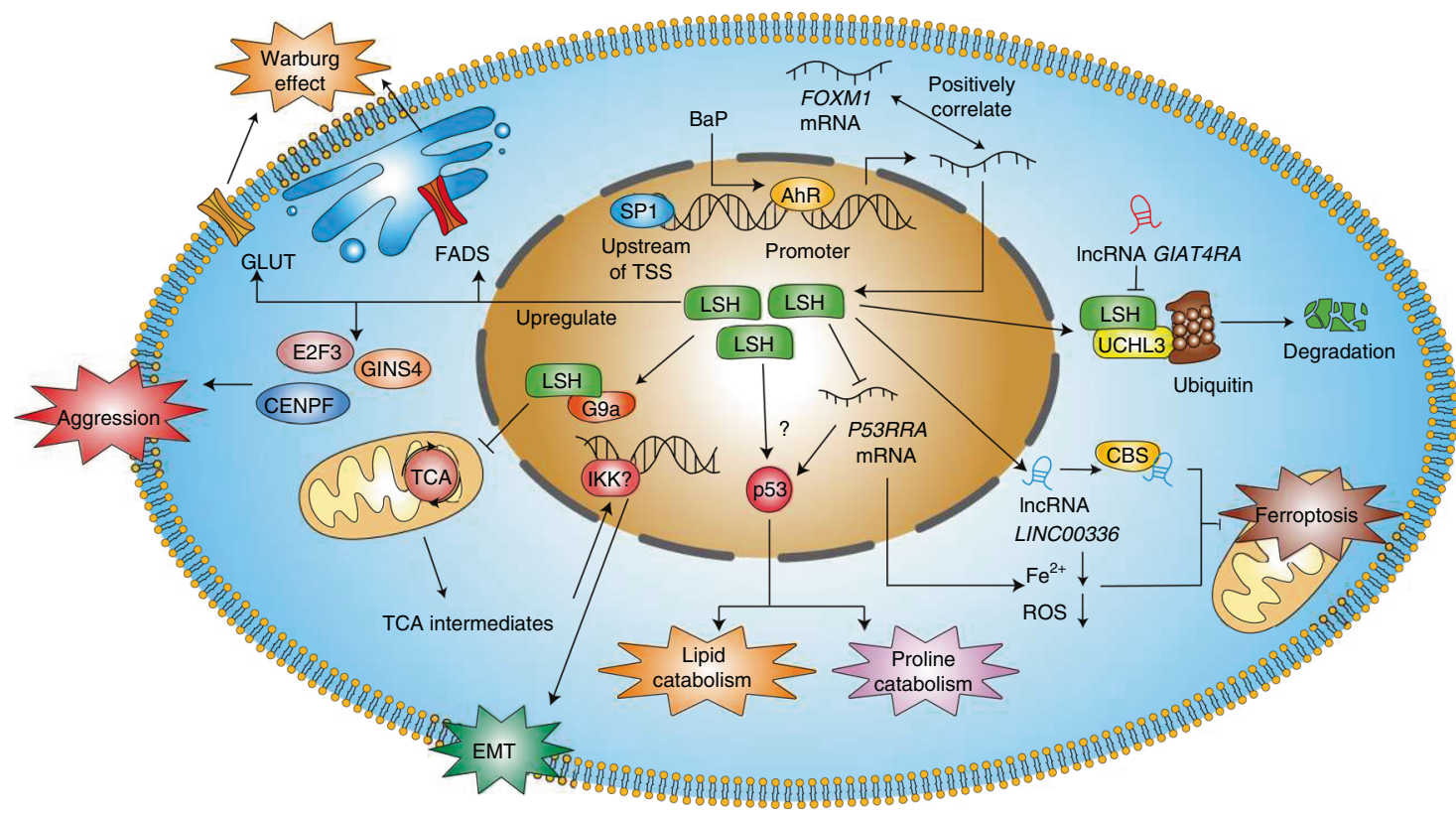

Fig. 3 The roles of LSH in carcinogenesis. LSH is elevated in many cancers, which results from the increase of oncogenic factors and the interference with its ubiquitination. AhR binds to the $L s h$ gene promotor, and SP1 assembles at the upstream of the TSS, elevating the levels of LSH. Besides, there is a positive correlation between FOXM1 and LSH mRNAs. The IncRNA GIAT4RA impedes LSH binding with UCHL3, therefore hinders the ubiquitination of LSH. Increased LSH impacts metabolism, progress and even the fate of cancerous cells. LSH upregulates GLUTs and FADSs, activates TP53 protein and curbs FH. As a consequence, it promotes the Warburg effect, engages in TP53-related lipid catabolism, promotes TP53-related proline catabolism, and suppresses TCA, which motivates IKK $\alpha$ to switch binding sites and consequently, to promote EMT. Increased levels of LSH increase the levels of oncogenic proteins, including GINS4, E2F3 and CENPF, preserving the aggressive phenotype. Also, it inhibits ferroptosis by increasing the levels of the IncRNA LINC00336 and decreasing P53RRA mRNA. The IncRNA LINC00336 stabilises CBS, enhancing cell resistance to ferroptosis. The decline of P53RRA mRNA downregulates Fe ${ }^{2+}$ and ROS, and as a result, inhibits ferroptosis. However, the relationship between TP53 and LSH needs a cleared elucidation since P53RRA mRNA upregulates TP53 in an upstream manner. In summary, the anomalous expression of LSH accelerates carcinogenesis and leads to worse clinical outcomes. 
SNF-rel (231aa-577aa)

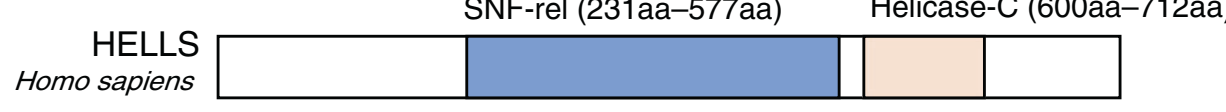

(838 aa)

SNF-rel (224aa-560aa) Helicase-C (583aa-695aa)

\begin{tabular}{|l|l|l|l|l|}
\hline LSH & & & & \\
\hline Mus musculus
\end{tabular}

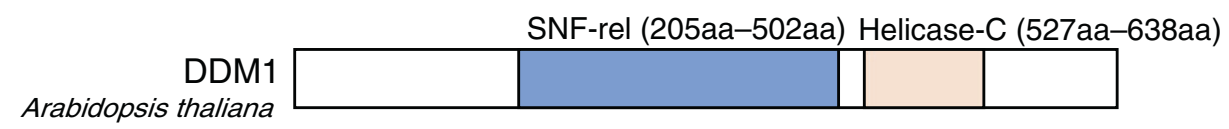

(764 aa)
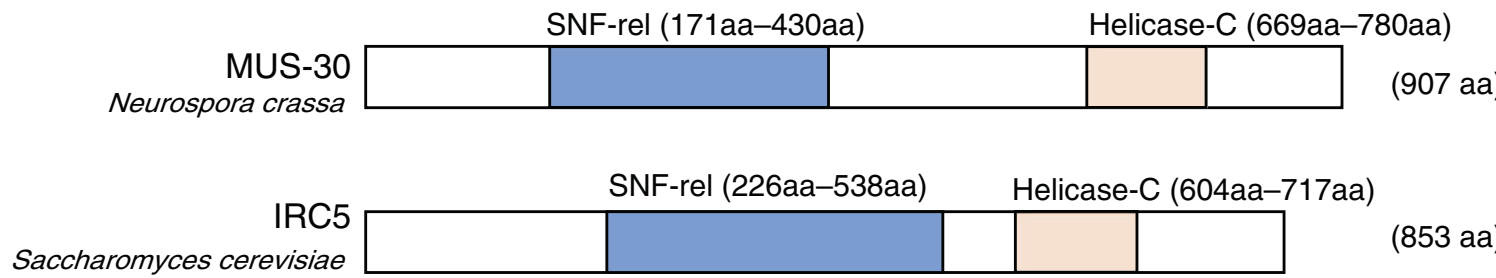

(853 aa)

Fig. 4 Domains of LSH homologs. Five homologs from animals, plants and fungi are depicted above. Protein names and species are listed at the left. Conserved domains are labelled. SNF-rel refers to SNF2 related domain; Helicase_C refers to Helicase conserved C-terminal domain.

addition, high levels of LSH correlates with a poor prognosis in gastric cancer [72]. The mechanism associated with this observation might act through reduced levels of miR-365a-3p, since this microRNA binds to its 3 ' untranslated region (UTR) of the HELLS transcript and represses its translation, thereby resulting in reduced LSH levels and enhanced carcinogenesis [73].

At the network level, LSH interacts with various modulators in different pathways, resulting in a more aggressive phenotype of tumour cells (Fig. 3). LSH controls the fate of tumour cells by its interaction with TP53 [70, 71, 74]. In nasopharyngeal cancer cells (NPCs), including HK1 and CNE1, there are at least three different mechanisms for LSH to induce activation of TP53, (i) by removing K11-linked and K48-linked polyubiquitin chain type in TP53; (ii) by interfering in the interaction between TP53 and MDM2 (a critical ubiquitin ligase of TP53); or (iii) by directly binding TP53 to activate TP53-mediated lipid catabolism, which in succession induces the expression of genes such as carnitine palmitoyltransferase $1 \mathrm{~B}$ and $1 \mathrm{C}$ (CPT1B and CPT1C) [71]. Besides being a positive regulator of TP53, the cooperation between LSH and TP53 is potentially more refined. For instance, in cell lines derived from human lungs, LSH epigenetically silences the expression of P53RRA, a tumour suppressor gene that acts as an upstream activator of TP53 and is involved in cell-cycle arrest, apoptosis and ferroptosis [70]. As discussed above, the precise impact of LSH on TP53 can be exerted by multiple mechanisms. Therefore, the conventional view concerning the balance between survival and death of tumour cells should be reconsidered.

In the regulation of metabolic pathways, the overexpression of Lsh inhibits ferroptosis, contributes to the Warburg effect, and promotes proline catabolism $[70,75,76]$. Concerning ferroptosis, LSH inhibits the expression of P53RRA [70], consequently decreasing two significant ferroptosis hallmarks, namely intracellular concentrations of $\mathrm{Fe}^{2+}$ and reactive oxygen species (ROS) [77]. LSH inhibits ferroptosis by increasing the expression of the IncRNA LINC00336 to preserve cystathionine- $\beta$-synthase (CBS) levels, which fosters erastin-induced ferroptosis resistance [78]. Regarding the Warburg effect, apart from indirect effects via TP53, LSH promotes the expression of glucose transporters (GLUT), including GLUT1, GLUT6, GLUT12 and GLUT13, as well as the expression of fatty acid desaturases (FADS) including FADS2 and FADS5, which is consistent with the involvement of LSH in the expression of metabolism-related genes as well as boosting the Warburg effect [75]. Moreover, the recruitment of LSH and the euchromatic histone-lysine $\mathrm{N}$-methyltransferase 2 (EHMT2), also known as G9a [79], to fumarate hydratase $(F H)$ promoters represses its expression. Further, it leads to increased TCA intermediates, such as $\mathrm{a}-\mathrm{KG}$ and citrate, which prompt the inhibitor of nuclear factor kappa-B kinase alpha (IKKa) to bind to the promoters of vimentin (VIM) and impair its binding to zonula occludens-1 (ZO-1) and E-cadherin, producing malignant progression [80]. As for proline catabolism, in NSCLC LSH facilitates TP53 binding to the promoter of proline dehydrogenase $(P R O D H)$ and elevates the levels of PRODH, which next fosters the expression of inflammatory cytokines including CXC11, LCN2 and IL17C [76].

Ectopic expression of $L s h$ promotes tumour growth. In HCC, LSH increases the levels of centromere protein $\mathrm{F}$ by binding to the transcription start site of its gene (CENPF), which positively correlates with tumour growth of xenografts in mice [67]. In colon cancer cells, LSH interacts with E2F transcription factor 3 (E2F3) in vivo and cooperates with its oncogenic functions [81]. Von Eys et al. identified genome-wide common target genes of LSH and E2F3 by chromatin immunoprecipitation followed by DNA sequencing [81]. Moreover, LSH binds and co-regulates promoters of active E2F3-target genes, including the trithoraxrelated MLL1. Besides, increased levels of LSH may promote epithelial-mesenchymal transition (EMT) [68]. In NSCLCs, the overexpression of Lsh boosts GINS4 expression, which decreases the epithelial marker E-cadherin (CDH1) and increases the mesenchymal markers VIM and SNAI1 [68, 75]. It is suggested that LSH directly interacts with the 3' UTR of the GINS4 mRNA, as confirmed by RNA immunoprecipitation assays [68].

However, in metastatic cancer cells, a modest decline of LSH has been observed [32]. Comparing healthy human tissues, nonmetastatic and metastatic tissues of NPC, colon and breast cancers, the protein levels of LSH and TET are statistically lowest in metastatic cancer tissues. Since TET converts 5-methylcytosine $(5 \mathrm{mC})$ to 5 -hmC, and LSH acts as a 5 - $\mathrm{hmC}$ reader in mouse embryonic stem cells $[82,83]$, it would not be surprising to observe a relative decline of $5-\mathrm{hmC}$ in metastatic cancer tissues in comparison to non-metastatic ones. Moreover, the decline of 5 -hmC correlates to genomic instability, which may explain the mild reduction of LSH in metastatic tissues [32].

In summary, the levels of LSH are elevated in many cancer types. More importantly, the vast amount of evidence indicates that LSH acts as a driver of malignant progression of tumour cells via its interaction with IncRNAs, oncoproteins or tumour 
suppressors, its primary control of transcriptional activity as a modulator of DNA methylation or its direct binding with posttranslationally modified proteins [84], consequently promote metabolic changes, EMT and tumour growth, as well as ferroptosis inhibition. However, a previous article reported a decrease in the expression of Lsh in metastatic cancers compared with nonmetastatic ones and healthy tissues, which evidences the need for further studies and more robust statistics to validate this finding [32]. Nevertheless, the increase of LSH is still tightly linked with poor clinical outcomes and is emerging as a novel prognostic marker [85].

\section{Homologs of LSH}

There are multiple helicase homologs of the SWI2/SNF2 family (Fig. 4). The most representative one is the protein decreases in DNA methylation 1 (DDM1) in plants. In alignment with LSH function, DDM1 controls DNA methylation [86]. DDM1 mainly contributes to the cytosine methylation of DNA, including $\mathrm{CpG}$, $\mathrm{CHG}(\mathrm{H}=\mathrm{A}, \mathrm{C}$ or $\mathrm{T})$, and $\mathrm{CHH}$ sequences in plants $[74,87]$. Knockout of DDM1 not only results in immediate hypomethylation at regions marked by $\mathrm{H} 3 \mathrm{~K} 9 \mathrm{me} 2$ but also leads to heritable epialleles in self-pollinated lines. For instance, the progressive loss of DNA methylation at differentially methylated regions with lower H3K9me2 marks at the flowering Wageningen (FWA) locus induces inheritable changes in late-flowering phenotype [88, 89]. During heterochromatin formation, the Arabidopsis nucleosome remodeler DDM1 facilitates the access of DNA methyltransferase DRM2 to H1-containing chromatin $[90,91]$. As a result, DDM1 inhibits small RNA-directed DNA methylation by suppressing the expression of small RNAs [87, 92]. Besides, DDM1 may control pressure responses via transposable elements and stress-related long intergenic noncoding RNAs [93].

In fungi, the LSH homolog induces different effects when compared to the mammalian or botanical ones. The Neurospora homolog mutagen sensitive-30 (MUS-30) contains an N-terminal SNF2 DEAD-box helicase domain and a C-terminal HelicC domain. In contrast with LSH or DDM1, MUS-30 exhibits no influence on DNA methylation or meiosis. However, a study verified that this kind of LSH/DDM1 homolog is essential during DNA damage. In detail, knockout of MUS-30 results in hypersensitivity to methyl methanesulfonate, and replication forks may collapse, resulting in double-strand breaks (DSB). Moreover, MUS-30 is necessary for toxic base-excision repair instead of general HR or NHEJ pathways [51].

The increased recombination centres 5 (IRC5) protein from Saccharomyces cerevisiae is a member of the conserved SNF2 family of ATP-dependent DNA translocases. Similarly, IRC5 plays a role in resistance to DNA damage. Epistasis analysis showed that the impaired expression of IRC5 disrupts the interaction between checkpoint activators of DNA damage and genes involved in DNAdamage tolerance. Furthermore, ICR5 is essential for cohesin binding to centromeres and chromosome arms. ICR5 interacts with genes that encode proteins mediating the replication fork stability and sister chromatid cohesion (SCC). Mutation of IrC5 leads to reduced SCC1 protein levels and impaired interaction between SCC1 and SCC2 proteins, consequently decreasing the levels of cohesin, causing separation of premature sister chromatid. Moreover, the mutant Irc5 contributes to the loss of rDNA repeats, undermining the cellular homoeostasis [94].

Overall, LSH and its homologs are guardians of genomic stability and protectors of the DNA structure. A similar contribution to DNA methylation by $\mathrm{LSH}$ and DDM1 may reveal a conserved mechanism between plants and mammals and could provide a distinctive insight into the functions of $\mathrm{LSH}[86,93]$. However, higher molecular diversity is present in fungi since MUS30 and IRC50, the homologs of $\mathrm{LSH}$, mainly monitor the structure of DNA and take part in chromatin structure but have shown little impact on DNA methylation [51, 94].

\section{CONCLUDING REMARKS}

LSH, along with its homologs, facilitates heterochromatin formation, epigenetic silencing of target genes and DNA-damage repair. A better characterisation of the roles of LSH in the mechanisms modulating chromatin structure, differentiation of stem cells and oncogenesis will be fundamental for further studies. More research is expected to reveal the detailed mechanisms of the interaction between LSH and the DNA methyltransferase DNMT3B and the DNA demethylating TET proteins. Besides, the general interest in the role of LSH in tumour cells has broadened. Apart from epigenetic modulations, LSH is substantially involved in the metabolism of tumour cells. Multilayered interactions between LSH and TP53 and TP53-relevant lipid catabolism demand more experiments to elucidate the exact role of LSH in metabolism [71]. Associated with the Warburg effect, ferroptosis and proline catabolism, LSH emerges as a potential target to suppress cancers' malignant progression. As indicated by recent studies, ectopics level of LSH may result in severe clinical outcomes [18]; on the contrary, the knockdown of LSH suppresses the migration of tumour cells [80]. Suppressors of LSH may be promising for clinical applications against the malignant progression of cancers, though more investigations are required to portray how LSH mediates metabolism and immunity and whether targeting LSH is a beneficial choice.

\section{Reporting summary}

Further information on experimental design is available in the Nature Research Reporting Summary linked to this paper.

\section{DATA AVAILABILITY}

Data analysed in Fig. 2 were extracted from the TCGA database (https://portal.gdc. cancer.gov/). The overall survival rate graph of NSCLC is from the study conducted by Mao et al., and the usage of the graph was approved (PMID: 30094095).

\section{REFERENCES}

1. Cavalli $G$, Heard $E$. Advances in epigenetics link genetics to the environment and disease. Nature. 2019:571:489-99.

2. Boland M, Nazor K, Loring J. Epigenetic regulation of pluripotency and differentiation. Circulation Res. 2014;115:311-24.

3. Dobersch, S, Rubio, K, Barreto, G. Pioneer factors and architectural proteins mediating embryonic expression signatures in cancer. Trends Mol Med. 2019. https://doi.org/10.1016/j.molmed.2019.01.008.

4. Singh AK, Mueller-Planitz F. Nucleosome positioning and spacing: from mechanism to function. J Mol Biol. 2021;433:166847.

5. Flaus A, Martin D, Barton G, Owen-Hughes T. Identification of multiple distinct Snf2 subfamilies with conserved structural motifs. Nucleic Acids Res. 2006;34:2887-905.

6. Bartholomew B. Regulating the chromatin landscape: structural and mechanistic perspectives. Annu Rev Biochem. 2014;83:671-96.

7. Ozturk N, Singh I, Mehta A, Braun T, Barreto G. HMGA proteins as modulators of chromatin structure during transcriptional activation. Front Cell Dev Biol. 2014;2:5.

8. Lee DW, Zhang K, Ning ZQ, Raabe EH, Tintner S, Wieland R, et al. Proliferationassociated SNF2-like gene (PASG): a SNF2 family member altered in leukemia. Cancer Res. 2000;60:3612-22.

9. Jarvis CD, Geiman T, Vila-Storm MP, Osipovich O, Akella U, Candeias S, et al. A novel putative helicase produced in early murine lymphocytes. Gene. 1996;169:203-7.

10. Geiman T, Tessarollo L, Anver M, Kopp J, Ward J, Muegge K. Lsh, a SNF2 family member, is required for normal murine development. Biochimica et Biophysica Acta. 2001;1526:211-20.

11. Dennis K, Fan T, Geiman T, Yan Q, Muegge K. Lsh, a member of the SNF2 family, is required for genome-wide methylation. Genes Dev. 2001;15:2940-4.

12. Yan $Q$, Huang J, Fan T, Zhu H, Muegge K. Lsh, a modulator of CpG methylation, is crucial for normal histone methylation. EMBO J. 2003;22:5154-62.

13. Muegge K, Geiman T, Xi S, Jiang Q, Schmidtman A, Chen T, et al. Lsh is involved in de novo methylation of DNA. Proc Am Assoc Cancer Res Annu Meet. 2006:47:541-541. 
14. Zocchi L, Mehta A, Wu SC, Wu J, Gu Y, Wang J, et al. Chromatin remodeling protein HELLS is critical for retinoblastoma tumor initiation and progression. Oncogenesis. 2020;9:25.

15. Sha K, Boyer LA. The chromatin signature of pluripotent cells (May 31, 2009), StemBook, ed. The Stem Cell Research Community, StemBook, https://doi.org/ 10.3824/stembook.1.45.1, http://www.stembook.org.

16. Delgado-Olguin P, Recillas-Targa F. Chromatin structure of pluripotent stem cells and induced pluripotent stem cells. Brief Funct Genomics. 2011;10:37-49.

17. Ren J, Briones V, Barbour S, Yu W, Han Y, Terashima M, et al. The ATP binding site of the chromatin remodeling homolog $L$ sh is required for nucleosome density and de novo DNA methylation at repeat sequences. Nucleic Acids Res. 2015;43:1444-55.

18. Law C-T, Wei L, Tsang FH-C, Chan CY-K, Xu IM-J, Lai RK-H, et al. HELLS regulates chromatin remodeling and epigenetic silencing of multiple tumor suppressor genes in human hepatocellular carcinoma. Hepatology. 2019;69:2013-30.

19. Deaton AM, Bird A. CpG islands and the regulation of transcription. Genes Dev. 2011;25:1010-22.

20. Mehta A, Dobersch S, Romero-Olmedo AJ, Barreto G. Epigenetics in lung cancer diagnosis and therapy. Cancer Metastasis Rev. 2015. https://doi.org/10.1007/ s10555-015-9563-3.

21. Smith Z, Meissner A. DNA methylation: roles in mammalian development. Nat Rev Genet. 2013;14:204-20.

22. Jenness C, Giunta S, Müller MM, Kimura H, Muir TW, Funabiki H. HELLS and CDCA7 comprise a bipartite nucleosome remodeling complex defective in ICF syndrome. Proc Natl Acad Sci USA. 2018;115:E876-e885.

23. Termanis A, Torrea N, Culley J, Kerr A, Ramsahoye B, Stancheva I. The SNF2 family ATPase LSH promotes cell-autonomous de novo DNA methylation in somatic cells. Nucleic Acids Res. 2016;44:7592-604.

24. De La Fuente R, Baumann C, Fan T, Schmidtmann A, Dobrinski I, Muegge K. Lsh is required for meiotic chromosome synapsis and retrotransposon silencing in female germ cells. Nat Cell Biol. 2006;8:1448-54.

25. Zeng W, Baumann C, Schmidtmann A, Honaramooz A, Tang L, Bondareva A, et al. Lymphoid-specific helicase (HELLS) is essential for meiotic progression in mouse spermatocytes. Biol Reprod. 2011;84:1235-41.

26. Fan T, Yan Q, Huang J, Austin S, Cho E, Ferris D, et al. Lsh-deficient murine embryonal fibroblasts show reduced proliferation with signs of abnormal mitosis. Cancer Res. 2003;63:4677-83.

27. Baumann C, Ma W, Wang X, Kandasamy MK, Viveiros MM, De La Fuente R. Helicase LSH/Hells regulates kinetochore function, histone $\mathrm{H} 3 / \mathrm{Thr} 3$ phosphorylation and centromere transcription during oocyte meiosis. Nat Commun. 2020;11:4486-4486.

28. Dunican DS, Cruickshanks HA, Suzuki M, Semple CA, Davey T, Arceci RJ, et al. Lsh regulates LTR retrotransposon repression independently of Dnmt3b function. Genome Biol. 2013;14:R146.

29. Barreto G, Schafer A, Marhold J, Stach D, Swaminathan SK, Handa V, et al. Gadd45a promotes epigenetic gene activation by repair-mediated DNA demethylation. Nature. 2007;445:671-5.

30. Wu X, Zhang Y. TET-mediated active DNA demethylation: mechanism, function and beyond. Nat Rev Genet. 2017;18:517-34.

31. Ross SE, Bogdanovic O. TET enzymes, DNA demethylation and pluripotency. Biochem Soc Trans. 2019;47:875-85.

32. Jia J, Shi Y, Chen L, Lai W, Yan B, Jiang Y, et al. Decrease in lymphoid specific helicase and 5-hydroxymethylcytosine is associated with metastasis and genome instability. Theranostics. 2017;7:3920-32.

33. van der Wijst $M$, Venkiteswaran $M$, Chen $H, X u$ G, Plösch $T$, Rots $M$. Local chromatin microenvironment determines DNMT activity: from DNA methyltransferase to DNA demethylase or DNA dehydroxymethylase. Epigenetics. 2015;10:671-6.

34. Wang Y, Fischle W, Cheung W, Jacobs S, Khorasanizadeh S, Allis CD. Beyond the double helix: writing and reading the histone code. Novartis Found Symp. 2004;259:3-17. discussion 17-21, 163-169.

35. Singh I, Ozturk N, Cordero J, Mehta A, Hasan D, Cosentino C, et al. High mobility group protein-mediated transcription requires DNA damage marker gammaH2AX. Cell Res. 2015;25:837-50.

36. Dobersch S, Rubio K, Singh I, Gunther S, Graumann J, Cordero J, et al. Positioning of nucleosomes containing gamma-H2AX precedes active DNA demethylation and transcription initiation. Nat Commun. 2021;12:1072.

37. Yu W, Briones V, Lister R, McIntosh C, Han Y, Lee EY, et al. CG hypomethylation in Lsh-/- mouse embryonic fibroblasts is associated with de novo H3K4me1 formation and altered cellular plasticity. Proc Natl Acad Sci USA. 2014;111:5890-5.

38. Ren J, Hathaway NA, Crabtree GR, Muegge K. Tethering of Lsh at the Oct4 locus promotes gene repression associated with epigenetic changes. Epigenetics. 2018;13:173-81.

39. Han $\mathrm{Y}$, Ren J, Lee $\mathrm{E}, \mathrm{Xu} \mathrm{X}, \mathrm{Yu} \mathrm{W}$, Muegge $\mathrm{K}$. Lsh/HELLS regulates self-renewal/ proliferation of neural stem/progenitor cells. Sci Rep. 2017;7:1136.
40. Sparmann A, van Lohuizen M. Polycomb silencers control cell fate, development and cancer. Nat Rev Cancer. 2006;6:846-56.

41. Viré E, Brenner C, Deplus R, Blanchon L, Fraga M, Didelot C, et al. The Polycomb group protein EZH2 directly controls DNA methylation. Nature. 2006;439:871-4.

42. Singh I, Contreras A, Cordero J, Rubio K, Dobersch S, Gunther S, et al. MiCEE is a ncRNA-protein complex that mediates epigenetic silencing and nucleolar organization. Nat Genet. 2018;50:990-1001.

43. Myant K, Stancheva I. LSH cooperates with DNA methyltransferases to repress transcription. Mol Cell Biol. 2008;28:215-26.

44. Tubbs A, Nussenzweig A. Endogenous DNA damage as a source of genomic instability in cancer. Cell. 2017;168:644-56.

45. Unoki M, Funabiki H, Velasco G, Francastel C, Sasaki H. CDCA7 and HELLS mutations undermine nonhomologous end joining in centromeric instability syndrome. J Clin Investig. 2019;129:78-92.

46. Aguilera A, Garcia-Muse T. Causes of genome instability. Annu Rev Genet. 2013;47:1-32.

47. Scully R, Panday A, Elango R, Willis N. DNA double-strand break repair-pathway choice in somatic mammalian cells. Nat Rev Mol Cell Biol. 2019;20:698-714.

48. Jackson SP. Sensing and repairing DNA double-strand breaks. Carcinogenesis. 2002;23:687-96.

49. Dimitrova N, de Lange T. Cell cycle-dependent role of MRN at dysfunctional telomeres: ATM signaling-dependent induction of nonhomologous end joining (NHEJ) in G1 and resection-mediated inhibition of NHEJ in G2. Mol Cell Biol. 2009;29:5552-63.

50. Mahaney B, Meek K, Lees-Miller S. Repair of ionizing radiation-induced DNA double-strand breaks by non-homologous end-joining. Biochemical J. 2009;417:639-50.

51. Basenko E, Kamei M, Ji L, Schmitz R, Lewis Z. The LSH/DDM1 homolog MUS-30 is required for genome stability, but not for DNA methylation in Neurospora crassa. PLoS Genet. 2016;12:e1005790.

52. He YF, Ren JK, Xu XP, Ni K, Schwader A, Finney R, et al. Lsh/HELLS is required for B lymphocyte development and immunoglobulin class switch recombination. Proc Natl Acad Sci USA. 2020;117:20100-8.

53. Kollárovič G, Topping C, Shaw E, Chambers A. The human HELLS chromatin remodelling protein promotes end resection to facilitate homologous recombination and contributes to DSB repair within heterochromatin. Nucleic Acids Res. 2020;48:1872-85.

54. Syed A, Tainer J. The MRE11-RAD50-NBS1 complex conducts the orchestration of damage signaling and outcomes to stress in DNA replication and repair. Annu Rev Biochem. 2018:87:263-94.

55. Sartori A, Lukas C, Coates J, Mistrik M, Fu S, Bartek J, et al. Human CtIP promotes DNA end resection. Nature. 2007;450:509-14.

56. Paull T. Mechanisms of ATM activation. Annu Rev Biochem. 2015;84:711-38.

57. Wu L, Luo K, Lou Z, Chen J. MDC1 regulates intra-S-phase checkpoint by targeting NBS1 to DNA double-strand breaks. Proc Natl Acad Sci USA. 2008;105:11200-5.

58. Li J, Stern DF. DNA damage regulates Chk2 association with chromatin. J Biol Chem. 2005;280:37948-56.

59. Kakarougkas A, Ismail A, Klement K, Goodarzi A, Conrad S, Freire R, et al. Opposing roles for 53BP1 during homologous recombination. Nucleic Acids Res. 2013;41:9719-31.

60. Burrage J, Termanis A, Geissner A, Myant K, Gordon K, Stancheva I. The SNF2 family ATPase LSH promotes phosphorylation of $\mathrm{H} 2 \mathrm{AX}$ and efficient repair of DNA double-strand breaks in mammalian cells. J cell Sci. 2012;125:5524-34.

61. Spruce C, Dlamini S, Ananda G, Bronkema N, Tian H, Paigen K, et al. HELLS and PRDM9 form a pioneer complex to open chromatin at meiotic recombination hot spots. Genes Dev. 2020;34:398-412.

62. Roos W, Krumm A. The multifaceted influence of histone deacetylases on DNA damage signalling and DNA repair. Nucleic Acids Res. 2016;44:10017-30.

63. Miller K, Tjeertes J, Coates J, Legube G, Polo S, Britton S, et al. Human HDAC1 and HDAC2 function in the DNA-damage response to promote DNA nonhomologous end-joining. Nat Struct Mol Biol. 2010;17:1144-51.

64. Johnson D, Spitz G, Tharkar S, Quayle S, Shearstone J, Jones S, et al. HDAC1,2 inhibition impairs EZH2- and BBAP-mediated DNA repair to overcome chemoresistance in EZH2 gain-of-function mutant diffuse large B-cell lymphoma. Oncotarget. 2015;6:4863-87.

65. Rubio K, Singh I, Dobersch S, Sarvari P, Gunther S, Cordero J, et al. Inactivation of nuclear histone deacetylases by EP300 disrupts the MiCEE complex in idiopathic pulmonary fibrosis. Nat Commun. 2019;10:2229.

66. Lee SJ, Jang H, Park C. Maspin increases Ku70 acetylation and Bax-mediated cell death in cancer cells. Int J Mol Med. 2012;29:225-30.

67. Yang R, Liu N, Chen L, Jiang Y, Shi Y, Mao C, et al. GIAT4RA functions as a tumor suppressor in non-small cell lung cancer by counteracting Uchl3-mediated deubiquitination of LSH. Oncogene. 2019;38:7133-45. 
68. Yang R, Liu N, Chen L, Jiang Y, Shi Y, Mao C, et al. LSH interacts with and stabilizes GINS4 transcript that promotes tumourigenesis in non-small cell lung cancer. J Exp Clin Cancer Res: CR. 2019;38:280.

69. Waseem A, Ali M, Odell E, Fortune F, Teh M. Downstream targets of FOXM1: CEP55 and HELLS are cancer progression markers of head and neck squamous cell carcinoma. Oral Oncol. 2010;46:536-42.

70. Mao C, Wang X, Liu Y, Wang M, Yan B, Jiang Y, et al. A G3BP1-interacting IncRNA promotes ferroptosis and apoptosis in cancer via nuclear sequestration of p53. Cancer Res. 2018;78:3484-96.

71. Chen L, Shi Y, Liu N, Wang Z, Yang R, Yan B, et al. DNA methylation modifier LSH inhibits p53 ubiquitination and transactivates p53 to promote lipid metabolism. Epigenetics Chromatin 2019;12:1-22.

72. Peng X, Sun J, Long Y, Xiao D, Zhou J, Tao Y, et al. The significance of HOXC11 and LSH in survival prediction in gastric adenocarcinoma. OncoTargets Ther. 2021;14:1517-29.

73. Yang R, Liu G, Han L, Qiu Y, Wang L, Wang M. MiR-365a-3p-mediated regulation of HELLS/GLUT1 axis suppresses aerobic glycolysis and gastric cancer growth. Front Oncol. 2021;11:616390.

74. Long J, Xia A, Liu J, Jing J, Wang Y, Qi C, et al. Decrease in DNA methylation 1 (DDM1) is required for the formation of $\mathrm{CHH}$ islands in maize. J Integr Plant Biol. 2019;61:749-64.

75. Jiang Y, Mao C, Yang R, Yan B, Shi Y, Liu X, et al. EGLN1/c-Myc induced lymphoidspecific helicase inhibits ferroptosis through lipid metabolic gene expression changes. Theranostics. 2017;7:3293-305.

76. Liu Y, Mao C, Wang M, Liu N, Ouyang L, Liu S, et al. Cancer progression is mediated by proline catabolism in non-small cell lung cancer. Oncogene. 2020;39:2358-76.

77. Dixon S, Stockwell B. The role of iron and reactive oxygen species in cell death Nat Chem Biol. 2014;10:9-17.

78. Liu, N, Lin, X, Huang, C. Activation of the reverse transsulfuration pathway through NRF2/CBS confers erastin-induced ferroptosis resistance. $\mathrm{Br} J$ Cancer. 2019. https://doi.org/10.1038/s41416-019-0660-x.

79. Myant K, Termanis A, Sundaram A, Boe T, Li C, Merusi C, et al. LSH and G9a/GLP complex are required for developmentally programmed DNA methylation. Genome Res. 2011;21:83-94.

80. He X, Yan B, Liu S, Jia J, Lai W, Xin X, et al. Chromatin remodeling factor LSH drives cancer progression by suppressing the activity of fumarate hydratase. Cancer Res. 2016;76:5743-55.

81. von Eyss B, Maaskola J, Memczak S, Möllmann K, Schuetz A, Loddenkemper C, et al. The SNF2-like helicase HELLS mediates E2F3-dependent transcription and cellular transformation. EMBO J. 2012;31:972-85.

82. Rawłuszko-Wieczorek A, Siera A, Jagodziński P. TET proteins in cancer: current 'state of the art'. Crit Rev Oncol/Hematol. 2015;96:425-36.

83. Spruijt C, Gnerlich F, Smits A, Pfaffeneder T, Jansen P, Bauer C, et al. Dynamic readers for 5-(hydroxy)methylcytosine and its oxidized derivatives. Cell. 2013;152:1146-59.

84. Liu N, Yang R, Shi Y, Chen L, Liu Y, Wang Z, et al. The cross-talk between methylation and phosphorylation in lymphoid-specific helicase drives cancer stem-like properties. Signal Transduct Target Ther. 2020;5:197.

85. Chen D, Maruschke M, Hakenberg O, Zimmermann W, Stief C, Buchner A. TOP2A HELLS, ATAD2, and TET3 are novel prognostic markers in renal cell carcinoma. Urology. 2017;102:265.e261-265.e267.

86. Yan X, Dong X, Liu L, Yang Y, Lai J, Guo Y. DNA methylation signature of intergenic region involves in nucleosome remodeler DDM1-mediated repression of aberrant gene transcriptional read-through. J Genet genomics $=$ Yi Chuan Xue Bao. 2016;43:513-23.

87. Corem $S$, Doron-Faigenboim A, Jouffroy $O$, Maumus $F$, Arazi $T$, Bouché $N$. ddm1Redistribution of $\mathrm{CHH}$ methylation and small interfering RNAs across the Genome of Tomato Mutants. Plant cell. 2018;30:1628-44.

88. Ito T, Tarutani Y, To T, Kassam M, Duvernois-Berthet E, Cortijo S, et al. Genomewide negative feedback drives transgenerational DNA methylation dynamics in Arabidopsis. PLoS Genet. 2015;11:e1005154

89. Kinoshita Y, Saze H, Kinoshita T, Miura A, Soppe W, Koornneef M, et al. Control of FWA gene silencing in Arabidopsis thaliana by SINE-related direct repeats. Plant J: Cell Mol Biol. 2007;49:38-45.

90. Zemach A, Kim M, Hsieh P, Coleman-Derr D, Eshed-Williams L, Thao K, et al. The Arabidopsis nucleosome remodeler DDM1 allows DNA methyltransferases to access H1-containing heterochromatin. Cell. 2013;153:193-205.

91. Lyons, D, Zilberman, D. DDM1 and Lsh remodelers allow methylation of DNA wrapped in nucleosomes. eLife. 2017;6:e30674.
92. Tan F, Lu Y, Jiang W, Wu T, Zhang R, Zhao $Y$, et al. DDM1 represses noncoding RNA expression and RNA-directed DNA methylation in heterochromatin. Plant Physiol. 2018;177:1187-97.

93. Wang D, Qu Z, Yang L, Zhang Q, Liu Z, Do T, et al. Transposable elements (TEs) contribute to stress-related long intergenic noncoding RNAs in plants. Plant J: Cell Mol Biol. 2017:90:133-46.

94. Litwin I, Bakowski T, Maciaszczyk-Dziubinska E, Wysocki R. The LSH/HELLS homolog Irc5 contributes to cohesin association with chromatin in yeast. Nucleic Acids Res. 2017;45:6404-16.

95. Mao C, Wang M, Qian B, Ouyang L, Shi Y, Liu N, et al. Aryl hydrocarbon receptor activated by benzo (a) pyrene promotes SMARCA6 expression in NSCLC. Am J Cancer Res. 2018;8:1214-27.

\section{ACKNOWLEDGEMENTS}

We thank all the members of the laboratory for their resourceful comments on the manuscript.

\section{AUTHOR CONTRIBUTIONS}

YT, DX and GB contributed to the conception of the study; XC, Yamei Li and KR wrote the manuscript; BD, Yuyi Li and QT performed the data analyses; CM and SL helped with constructive discussions.

\section{FUNDING INFORMATION}

This work was supported by the National Natural Science Foundation of China [82072594, 81672787, YT; 82073097, 81874139, 81672991, S.Liu; 82002916, CM 82073136, 81772927, DX], China Postdoctoral Science Foundation [2019 M652804, CM], Natural Science Foundation of Hunan Province [2020JJ5790, CM], Hunan Provincial Key Area R\&D Programs [2019SK2253, YT], Postdoctoral Foundation of Central South University [220372, CM], Shenzhen Science and Technology Program [KQTD20170810160226082, YT], and Shenzhen Municipal Government of China [JCYJ20180507184647104, YT]. Guillermo Barreto was funded by the "Université ParisEst Créteil" (UPEC, Créteil, France), the "Centre National de la Recherche Scientifique" (CNRS, France), "Délégation Centre-Est" (CNRS-DR6), the "Lorraine Université" (LU, France) through the initiative "Lorraine Université d'Excellence" (LUE) and the dispositive "Future Leader" and the "Deutsche Forschungsgemeinschaft" (DFG, Bonn, Germany) (BA 4036/4-1). Karla Rubio was funded by the "Consejo de Ciencia y Tecnología del Estado de Puebla" (CONCYTEP, Puebla, Mexico) through the initiative International Laboratory EPIGEN.

\section{ETHICS APPROVAL AND CONSENT TO PARTICIPATE}

This work does not require any ethical approval or participating consent.

\section{CONSENT TO PUBLISH}

Not applicable.

\section{COMPETING INTERESTS}

The authors declare no competing interests. This manuscript has been read and approved by all the authors, and has not been submitted to or is not under consideration for publication elsewhere.

\section{ADDITIONAL INFORMATION}

Supplementary information The online version contains supplementary material available at https://doi.org/10.1038/s41416-021-01543-2.

Correspondence and requests for materials should be addressed to Desheng Xiao, Guillermo Barreto or Yongguang Tao.

Reprints and permission information is available at http://www.nature.com/ reprints

Publisher's note Springer Nature remains neutral with regard to jurisdictional claims in published maps and institutional affiliations. 